\title{
Utilidad de la castración para mejorar el engrasamiento de la canal y la calidad de la carne de añojos de raza Tudanca acabados en pastoreo
}

\author{
Serrano, E. ${ }^{\circledR} ;$ Humada, M.J. ${ }^{1}$ y Castrillo, B.
}

'CIFA. Gobierno de Cantabria. Muriedas. Cantabria.

PALABRAS ClaVe ADICIONALES

Cebón.

Terneza.

Color.

Grasa subcutánea.

Grasa intramuscular.

Raza rústica.

\section{ADDITIONAL KEYWORDS}

Castrated bull.

Tenderness.

Colour.

Subcutaneous fat.

Intramuscular fat.

Rustic breed

\section{INFORMACIÓN}

Cronología del artículo.

Recibido/Received: 23.11.2015

Aceptado/Accepted: 18.02.2016

On-line: 11.06 .2016

Correspondencia a los autores/Contact e-mail:

emmaserrano@cifacantabria.org

\section{RESUMEN}

Se utilizaron 10 terneros de raza Tudanca para estudiar el efecto de la castración a los 10 meses de edad. Desde los 14 hasta los 18 meses, sacrificio, los animales se acabaron en pastoreo. La ganancia media diaria de peso vivo desde la castración hasta el inicio del pastoreo fue menor en el grupo castrados (349 vs. $595 \mathrm{~g} /$ animal $/$ día; $p \leq 0,05)$, en la etapa de pastoreo las diferencias no fueron significativas $(755 \mathrm{vs}$. $904 \mathrm{~g} /$ animal $/$ día; $\mathrm{p}=0,159)$. El lote castrados presentó valores inferiores $(p \leq 0,05)$ de peso vivo al sacrificio, canal fría $(168$ vs. $202 \mathrm{~kg}$ ) y rendimiento canal, y superiores $(p \leq 0,05)$ de $\%$ de grasa diseccionable total de la $6^{a}$ costilla $(13,4$ vs. $8,6 \%)$ e intramuscular $(3,8$ vs. $2,1 \%)$. No se observaron diferencias $(p>0,1)$ en la conformación y engrasamiento de la canal. La carne de los animales castrados presentó a las 24 horas post-sacrificio valores superiores $(p \leq 0,05)$ de $C^{*}$ y una tendencia $(p \leq 0,1)$ a valores superiores de $H^{\circ}$. El lote castrados presentó valores inferiores $(p \leq 0,05)$ de fuerza máxima de corte $(4,1 \mathrm{vs} .6,3 \mathrm{~kg})$ a los 7 días de maduración de la carne.

\section{Usefulness of castration to enhance carcass famess and meat quality of grass finished Tudanca bulls}

\section{SUMMARY}

Ten Tudanca calves were used to study the effect of castration at 10 months of age. Since 14 months of age until slaughter, at 18 months of age, they remained on pasture. Average daily live weight gain since castration until turn-out to pasture were lower in the castrated group (349 vs. $595 \mathrm{~g} /$ animal/day; $\mathrm{p} \leq 0.05$ ) but differences in average daily gain were not significant during the following grazing period $(755 \mathrm{vs} .904 \mathrm{~g} / \mathrm{animal} /$ day; $p=0.159)$. The castrated group showed lower values $(p \leq 0.05)$ of live weight at slaughter, carcass weight (168 vs. $202 \mathrm{~kg})$, and dressing percentage, and higher $(p \leq 0.05)$ percentage of dissectionable total fat of the 6th rib $(13.4 \mathrm{vs} .8 .6 \%)$ and percentage of intramuscular fat (3.8 vs. $2.1 \%)$. No differences were observed between treatments $(p>0,1)$ on conformation and fatness carcass scores. Meat from castrated animals showed 24 hours post-slaughter higher values $(p \leq 0.05)$ of $C^{*}$ and a tendency $(p \leq 0.1)$ to higher values of $\mathrm{H}^{\circ}$. Meat from castrated animals showed lower values $(\mathrm{p} \leq 0.05)$ of maximum shear force (4.1 vs. $6.3 \mathrm{~kg})$ at 7 days of ageing.

\section{INTRODUCCIÓN}

La Tudanca es una raza autóctona de Cantabria clasificada en peligro de extinción (R.D. 2129/2008). Sus bajos rendimientos cárnicos y su capacidad para la deposición de grasa, en la canal y en la no canal, podrían desaconsejar el acabado en sistemas intensivos, $\mathrm{y}$ hacen posible el acabado en sistemas semiextensivos en pastoreo con aportes bajos de concentrado (Humada et al., 2013). Las canales de los animales acabados en pastoreo y/o con aportes limitados de concentrado se ven en muchas ocasiones penalizadas por presentar notas de engrasamiento inferiores a las de los acabados en sistemas más intensivos (Casasús et al., 2011).
La castración incrementa la deposición de grasa en la canal y en la carne y la terneza, pero afecta negativamente al ritmo de crecimiento (Crouse, 1983; Knight et al., 1999 y 2000b). El objetivo de este trabajo es estudiar el efecto de la castración a los 10 meses de edad sobre parámetros productivos, la calidad de la canal y de la carne de añojos y cebones (18 meses de edad) de raza Tudanca acabados en pastoreo.

\section{MATERIAL Y MÉTODOS}

La fase de producción se desarrolló en la finca Aranda del Gobierno de Cantabria $\left(43^{\circ} 23^{\prime} 15^{\prime \prime} \mathrm{N}, 04^{\circ} 11^{\prime} 32^{\prime \prime} \mathrm{W}\right.$; 
84 m s.n.m.). Se utilizaron 10 terneros nacidos en la paridera de invierno, destetados a los 6,5 meses de edad. Después del destete continuaron en pastoreo hasta el 11 de octubre. En esta fecha se estabularon y se alimentaron con silo de hierba a libre disposición y 1,5 kg/animal/día de concentrado comercial hasta la salida al pasto el 21 de marzo. El 22 de noviembre se castraron con una pinza de Burdizzo 5 terneros. Los animales se asignaron a uno de los dos lotes (castrados y enteros) considerando la fecha de nacimiento, el peso vivo y el semental del que procedían (tabla I). A partir del 21 de marzo (13,8 meses \pm 21 días de edad) los terneros permanecieron en pastoreo rotacional en dos parcelas con pasto natural de 1,67 y 1,37 ha y se suplementaron con $1,6 \mathrm{~kg}$ de harina de cebada y $1 \mathrm{~kg}$ de pulpa de remolacha deshidratada por animal y día hasta el sacrificio a los 18 meses de edad. La altura del pasto se midió 1 vez por semana con el objetivo de que la altura media de la hierba disponible no estuviese por debajo de $7 \mathrm{~cm}$. Se muestreó el pasto en oferta al entrar los animales en las parcelas tomando submuestras de unos $50 \mathrm{~g}$ cortadas a $4 \mathrm{~cm}$ del suelo en 50 puntos. Las muestras de pasto y las muestras del resto de alimentos (concentrado comercial, cebada, silo de hierba y pulpa de remolacha; 1 muestra de cada alimento mezcla de las muestras obtenidas una vez por semana) se analizaron para determinar su contenido en materia seca, proteína bruta, extracto etéreo, cenizas (AOAC, 1990), fibra bruta, fibra neutro detergente y fibra ácido detergente (Van Soest et al., 1991). El contenido en energía neta (UFL/kg MS) de todos los alimentos y el valor lastre de los forrajes (BBU/kg MS) se calculó según las ecuaciones del INRA (2007) (tabla II).

Los animales se pesaron individualmente cada 15 días. Los sacrificios se realizaron el 11 de julio (2 castrados y 2 enteros) y el 1 de agosto (3 castrados y 3 enteros). El pastoreo tuvo una duración media de 125 días. Se registró el peso de la grasa perirrenal y de la canal fría y las notas de conformación y engrasamiento (Reglamento (CE) 1183/2006). Las canales permanecieron en oreo a temperatura ambiente (entre 10 y $13^{\circ} \mathrm{C}$ ) hasta las 7 horas postsacrificio. A las $24 \mathrm{~h}$ del sacrificio, se midió el $\mathrm{pH}$ del músculo Longissimus dorsi entre la $4^{\mathrm{a}}$ y la $5^{\mathrm{a}}$ vértebra lumbar, se pesó el chuletero y se extrajo la chuleta de la $6^{a}$ costilla, que se utilizó para determinar la composición tisular mediante disección. El músculo Longissimus dorsi de la $6^{\circ}$ costilla se usó para determinar la composición química según los Métodos de Análisis de Productos Cár- nicos, BOE de 29 de agosto de 1979. Se extrajo el músculo Longissimus dorsi del chuletero restante y se cortaron 5 filetes de $2,5 \mathrm{~cm}$ de espesor que se utilizaron para determinar los parámetros CIE $\mathrm{L}^{*}$, $\mathrm{a}^{*}, \mathrm{~b}^{*}$ de color ( 3 filetes) a las 24 horas, 7 y 14 días postmortem (24h de maduración en canal más 6 o 13 días de maduración al vacío a $4^{\circ}$ C) y la resistencia al corte (2 filetes). Los parámetros de color $\mathrm{L}^{*}$ (luminosidad), $\mathrm{a}^{*}$ (índice de rojo), b* (índice de amarillo) se midieron utilizando un colorímetro Minolta CR-400 (iluminante D65, observador $2^{\circ}$ ). Las muestras se dejaron oxigenar durante 1 hora a $4^{\circ} \mathrm{C}$ envueltas con film permeable al oxígeno. Los valores de $\mathrm{C}^{*}$ (croma) y $\mathrm{H}^{\mathrm{o}}$ (tono) se calcularon $\operatorname{como} \mathrm{C}_{\mathrm{a}, \mathrm{b}}^{*}=\left[\left(\mathrm{a}^{*}\right)^{2}+\left(\mathrm{b}^{*}\right)^{2}\right]^{0,5} \mathrm{y} \mathrm{H}_{\mathrm{a}, \mathrm{b}}^{\mathrm{o}}=\arctan \left(\mathrm{b}^{*} / \mathrm{a}^{*}\right)$. La resistencia al corte se determinó en muestras cocidas en un baño de agua hasta alcanzar una $\mathrm{T}^{\mathrm{a}}$ interna de $70^{\circ} \mathrm{C}$ usando un texturómetro TA.XT plus con una sonda Warner-Braztler.

Los datos productivos y las características de la canal se compararon mediante un análisis de covarianza (efecto fijo: castración; covariable: peso vivo a la castración (eliminada del modelo cuando $\mathrm{p}>0,05)$ ). La composición tisular, el $\mathrm{pH}$ y la composición química de la carne se compararon mediante un análisis de varianza considerando la castración como factor de variación. Los parámetros de color y la resistencia al corte se analizaron utilizando un modelo de medidas repetidas (factores: castración, tiempo de maduración y su interacción). Todos los análisis se realizaron utilizando el procedimiento GLM del programa SPSS 17.0 (2008). Cuando $\mathrm{p}<0,1$ para el factor castración, se separaron las medias dentro de cada tiempo de maduración utilizando el test de Bonferroni.

\section{RESULTADOS Y DISCUSIÓN}

El consumo de alimentos concentrados por animal fue de 243, 210 y $140 \mathrm{~kg}$ de pienso, harina de cebada y pulpa de remolacha, respectivamente. Varios autores han observado una disminución en el ritmo de crecimiento como consecuencia de la castración (Knight et al., 2000b; Mach et al., 2009; Sanz et al., 2011) debida a la disminución de la producción de hormonas anabolizantes en los testículos y al estrés postraumático (Knight et al., 2000b; Mach et al., 2009). Coincidiendo con Knight et al. (1999), las diferencias significativas en el ritmo de crecimiento en el periodo de 4 meses post-castración ( $p=0,002$, tabla III) desaparecieron el periodo posterior de pastoreo $(\mathrm{p}=$

\begin{tabular}{|c|c|c|c|}
\hline & Castrados $(n=5)$ & Enteros $(n=5)$ & Significación \\
\hline Fecha de nacimiento & $2 / 2 \pm 29,9$ & $31 / 1 \pm 17,3$ & - \\
\hline Edad de castración (días) & $294 \pm 30,0$ & $295 \pm 17,3$ & n.s. ${ }^{1}$ \\
\hline Peso vivo a la castración (kg) & $201 \pm 37,5$ & $207 \pm 20,4$ & n.s. ${ }^{1}$ \\
\hline
\end{tabular}


Tabla II. Porcentaje de materia seca, composición química, contenido en energía neta de los alimentos (Percentage of dry matter, chemical composition and net energy content of feeds and forage ingestibility).

\begin{tabular}{|c|c|c|c|c|c|}
\hline & Silo de hierba & Concentrado comercial & Pasto & Cebada & Pulpa de Remolacha \\
\hline MS (\%) & 47,3 & 89,2 & $17,6(12,5-25,0)$ & 90,2 & 89,9 \\
\hline PB (\%MS) & 15,9 & 19,3 & $16,7(13,2-21,3)$ & 10,6 & 10,6 \\
\hline $\mathrm{EE}(\% \mathrm{MS})$ & 2,5 & 4,0 & $2,6(1,6-3,7)$ & 1,3 & 0,9 \\
\hline FB (\%MS) & 28,1 & 6,5 & $22,0(18,9-25,8)$ & 4,9 & 21,5 \\
\hline FAD (\%MS) & 34,2 & - & $25,9(21,4-30,1)$ & - & - \\
\hline FND (\%MS) & 57,1 & 23,0 & $51,0(42,5-59,2)$ & 24,6 & 47,6 \\
\hline Cenizas (\%MS) & 11,7 & 5,7 & $9,9(7,9-12,4)$ & 2,6 & 9,7 \\
\hline Energía neta (UFL/kg MS) & 0,67 & 1,1 & $0,97(0,84-1,0)$ & 1,16 & 0,99 \\
\hline Unidades lastre (ULB $\left.{ }^{10} / \mathrm{kg} \mathrm{MS}\right)$ & 1,13 & - & $0,91(0,84-1,2)$ & - & - \\
\hline
\end{tabular}

El concentrado comercial estaba constituido por las siguientes materias primas: maíz, harina de soja tostada, cebada, cascarilla de soja, proteína de granos de destilería de cebada, melaza de caña, suero dulce, suero reengrasado, carbonato cálcico, fosfato bicálcico y cloruro sódico. En el pasto fueron analizadas 11 muestras desde el 21/3 hasta el 31/7. MS= materia seca; $\mathrm{PB}=$ proteína bruta; $\mathrm{EE}=$ extracto etéreo; $\mathrm{FB}=$ fibra bruta; $\mathrm{FAD}=$ fibra ácido detergente; $\mathrm{FND}$ = fibra neutro detergente; $\mathrm{UFL}=$ energía neta para el mantenimiento y el crecimiento expresada en unidades forrajeras leche; ULB= ingestibilidad expresada en unidades lastre bovino.

0,159). Como consecuencia de las diferencias en el ritmo de crecimiento, los animales castrados presentaron un menor peso de canal $(p=0,005$, tabla III). Coincidiendo con Sanz et al. (2011), los animales castrados presentaron un menor rendimiento canal $(p=0,019)$. Mientras que Sanz et al. (2011) observaron un efecto negativo de la castración sobre la conformación de la canal, Mach et al. (2009), coincidiendo con los resultados obtenidos $(p=0,394)$, no observaron un efecto de la castración sobre este parámetro. A diferencia de lo observado por Knight et al. (1999), Mach et al. (2009) y Sanz et al. (2011), en el presente trabajo no se observó un efecto de la castración sobre la nota de engrasamiento $(p=0,347)$. Este resultado es coherente con los resultados de la disección de la $6^{\mathrm{a}}$ costilla. Así, la castración incrementó el \% de grasa total diseccionable $(\mathrm{p}=0,006)$, pero cuando se consideran separadamente la grasa intermuscular y subcutánea se observa que, en el caso de los castrados, este incremento se debe sólo a la grasa intermuscular $(p=0,002)$. El lote castrados presentó una tendencia $(p=0,06)$ a valores superiores de peso de la grasa perirrenal.

De acuerdo con los resultados obtenidos, ni Mach et al. (2009), ni Sanz et al. (2011), ni Monteiro

Tabla III. Efecto de la castración a los 10 meses de edad en los rendimientos productivos, las características de la canal y de la carne de añojos y cebones de raza Tudanca sacrificados a los 18 meses de edad (Effect of castration at 10 months of age on animal performance, carcass and meat characteristics of 18 months old Tudanca bulls)

\begin{tabular}{|c|c|c|c|c|c|}
\hline & Castrados $n=5$ & Enteros $\mathrm{n}=5$ & Desviación estándar & Valor $p$ & Valor $p$ covariable $^{1}$ \\
\hline GMD castración²-inicio pastoreo ${ }^{3}$ (g/día) & 349,2 & 595,4 & 89,08 & 0,002 & 0,521 \\
\hline GMD inicio pastoreo-sacrificio (g/día) & 755,4 & 904,2 & 151,31 & 0,159 & 0,802 \\
\hline Edad de sacrificio (días) & 537,4 & 538,8 & 19,22 & 0,911 & - \\
\hline Peso vivo sacrificio $(\mathrm{kg})$ & 326,6 & 375,2 & 27,30 & 0,013 & 0,047 \\
\hline Peso canal fría (kg) & 168,2 & 202,4 & 17,39 & 0,005 & 0,022 \\
\hline Rendimiento canal (\%) & 51,4 & 53,9 & 1,51 & 0,019 & 0,050 \\
\hline Conformación (1-18) & 3,2 & 3,6 & 0,71 & 0,394 & 0,028 \\
\hline Engrasamiento (1-15) & 5,0 & 4,6 & 0,63 & 0,347 & 0,130 \\
\hline Peso del chuletero ${ }^{4}(\mathrm{~kg})$ & 12,8 & 14,8 & 1,53 & 0,054 & 0,043 \\
\hline Peso de la grasa perirenal (g) & 3263 & 2083 & 852,5 & 0,060 & 0,816 \\
\hline Grasa diseccionable de la $6^{\text {a }}$ costilla (\%): & 13,3 & 8,6 & 2,02 & 0,006 & - \\
\hline Grasa diseccionable intermuscular & 11,3 & 7,2 & 1,45 & 0,002 & - \\
\hline Grasa diseccionable subcutánea & 2,0 & 1,4 & 0,74 & 0,209 & - \\
\hline pH 24 h postsacrificio & 5,5 & 5,7 & 0,18 & 0,198 & - \\
\hline Grasa intramuscular ms. Longissimus dorsi (\%) & 3,8 & 2,1 & 0,93 & 0,019 & - \\
\hline
\end{tabular}

1: covariable: peso vivo a la castración; GMD: ganancia media diaria; ${ }^{2}$ : castración a los 10 meses de edad; 3: inicio del pastoreo a los 14 meses de edad; ${ }^{4}$ : peso del chuletero desde la 6 costilla hasta el final del lomo bajo. 
et al. (2014), observaron un efecto de la castración sobre el $\mathrm{pH}$ final de la carne $(\mathrm{p}=0,198)$. Igualmente, de acuerdo con Knight et al. (1999) y Mach et al. (2009), la castración incrementó el contenido en grasa intramuscular $(p=0,019)$. Knight et al. (1999) y Mach et al. (2009) observaron un efecto positivo de la castración sobre la luminosidad $\left(\mathrm{L}^{*}\right)$, pero por el contrario, de acuerdo con nuestros resultados, Monteiro et al. (2014) no observaron un efecto de la castración sobre este parámetro ( $\mathrm{p}=0,144$, tabla IV). Knight et al. (1999) y Mach et al. (2009) registraron valores más altos de $\mathrm{a}^{*} \mathrm{y} \mathrm{b}^{*}$ en los animales castrados. En el presente trabajo se observaron valores medios más altos de los parámetros $\mathrm{H}^{\circ}$ y $\mathrm{C}^{*}$, aunque sólo se observaron diferencias significativas $(p<0,05)$ a las 24 horas y a los 14 días postsacrificio en el parámetro $C^{*}$ (figura 1). Se considera que valores de $C^{*}$ inferiores a 18 marcan el final de la vida útil de la carne (McDougall, 1982). Todos los valores se situaron por encima de este umbral. El parámetro $\mathrm{H}^{\circ}$ representa el color percibido por el consumidor, valores altos indican un desplazamiento del color hacia el amarillo. Los valores obtenidos fueron relativamente bajos, indicando una elevada estabilidad del color. Humada et al. (2014) observaron que la alimentación con pasto favorecía la acumulación de vitamina E, lo que disminuía la oxidación de los lípidos e incrementaba la estabilidad del color.

Knight et al. (1999) y Monteiro et al. (2014) no observaron diferencias entre castrados y enteros en la resistencia al corte; por el contrario, Mach et al. (2009) y Sanz et al. (2011) sí observaron un efecto positivo sobre este parámetro. En el presente trabajo, se observó una tendencia a un efecto de la castración sobre la resistencia al corte $(p=0,077$, tabla IV), siendo inferiores los valores a los 7 días de maduración en el lote castrados ( $\mathrm{p}=0,04$, figura 2). Estas diferencias desaparecieron a los 14 días $(\mathrm{p}=0,32)$. Brandstetter et al. (1998) observaron que la castración producía una reducción en el tamaño de las fibras musculares y un incremento en el número de fibras de contracción rápida, parámetros asociados con una mayor terneza y una mayor velocidad de maduración de la carne
Figura 1. Efecto de la castración a los 10 meses de edad sobre los parámetros de color luminosidad $\left(\mathrm{L}^{*}\right)$, tono $\left(\mathrm{H}^{\circ}\right)$ y saturación $\left(\mathrm{C}^{*}\right)$ a los 1,7 y 14 días de maduración de la carne de añojos y cebones de raza Tudanca sacrificados a los 18 meses de edad (Effect of castration at 10 months of age on meat lightness $\left(\mathrm{L}^{*}\right)$, hue angle $\left(\mathrm{H}^{\circ}\right)$ and chroma $\left(\mathrm{C}^{*}\right)$ at 1,7 and 14 days of aging time of 18 months old Tudanca Bulls)
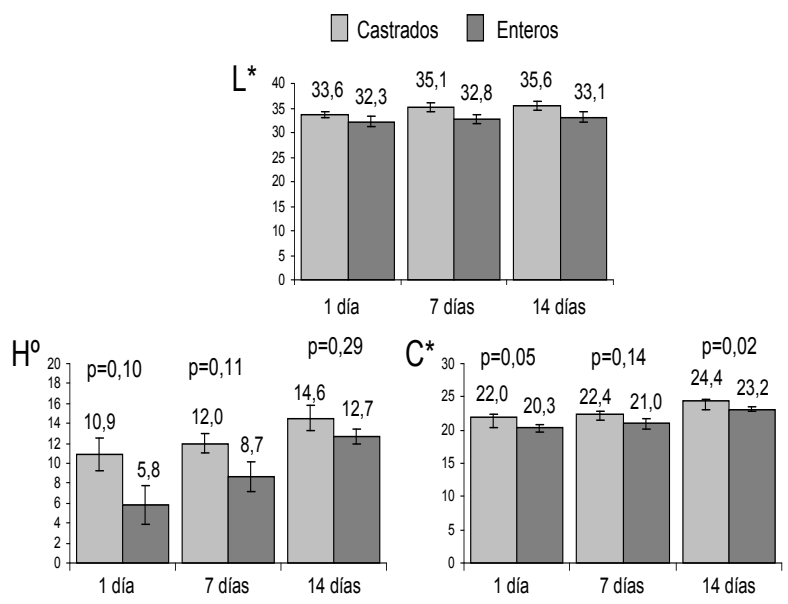

Figura 2. Efecto de la castración a los 10 meses de edad sobre la fuerza máxima de corte a los 7 y 14 días de maduración de la carne de añojos y cebones de raza Tudanca sacrificados a los 18 meses de edad (Effect of castration at 10 month of age on maximum shear force meat values at 7 and 14 days of aging time of 18 months old Tudanca Bulls).

Castrados

Enteros

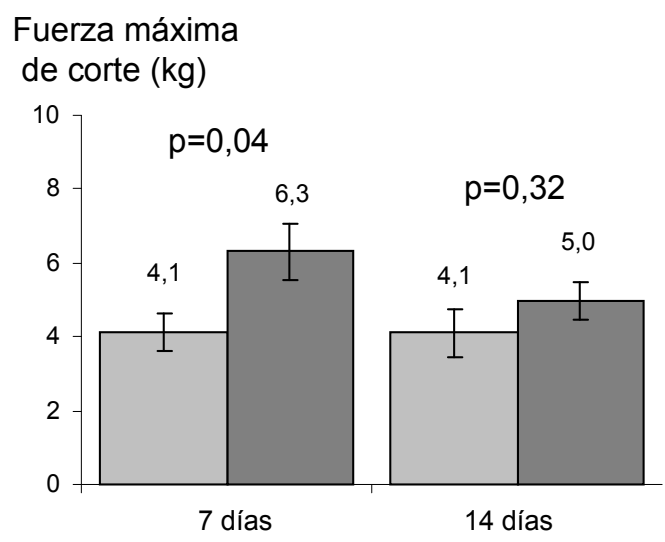

Tabla IV. Efecto de la castración a los 10 meses de edad (C; castrados vs. enteros) y del tiempo de maduración (M; 1, 7 o 14 días para los parámetros de color y 7 o 14 para la fuerza de corte) sobre los valores de luminosidad $\left(\mathrm{L}^{*}\right)$, tono $\left(\mathrm{H}^{\circ}\right)$, saturación $\left(\mathrm{C}^{*}\right)$ y fuerza máxima de corte (Fmax) de la carne de añojos y cebones de raza Tudanca sacrificados a los 18 meses de edad (Effect of castration at 10 months of age (C; castrated vs. entire) and aging time $\left(\mathrm{M} ; 1,7\right.$ or 14 days) on meat lightness $\left(\mathrm{L}^{*}\right)$, hue angle $\left(\mathrm{H}^{\circ}\right)$, chroma $\left(\mathrm{C}^{*}\right)$ and maximum shear force values $(\mathrm{Fmax})$ of 18 months old Tudanca bulls)

\begin{tabular}{|c|c|c|c|c|c|c|c|c|}
\hline \multirow[b]{2}{*}{ Factores } & \multicolumn{2}{|c|}{$L^{*}$} & \multicolumn{2}{|c|}{$\mathrm{H}^{\circ}$} & \multicolumn{2}{|c|}{$C^{*}$} & \multicolumn{2}{|c|}{ Fmax } \\
\hline & $\mathrm{F}$ & Sig. & $\mathrm{F}$ & Sig. & $\mathrm{F}$ & Sig. & $\mathrm{F}$ & Sig. \\
\hline Castración & 2,62 & 0,144 & 6,23 & 0,041 & 13,62 & 0,008 & 4,12 & 0,077 \\
\hline Maduración & 19,34 & 0,000 & 7,54 & 0,020 & 16,98 & 0,003 & 2,29 & 0,168 \\
\hline$C \times M$ & 3,27 & 0,641 & 0,70 & 0,460 & 0,10 & 0,791 & 2,21 & 0,176 \\
\hline
\end{tabular}


(Ouali, 1992). Los valores de resistencia al corte de los dos lotes fueron relativamente bajos, a ello puede haber contribuido el protocolo de refrigeración aplicado (oreo a $\mathrm{T}^{\mathrm{a}}$ ambiente durante 7 horas) (Crouse et al., 1983), pero incluso en estas condiciones, la castración mostró un efecto positivo sobre la terneza.

\section{AGRADECIMIENTOS}

César Cimadevilla y Nahum Chomón, Personal de la Finca Aranda y del Laboratorio Agrícola del CIFA. Servicio de Laboratorio y Control del Gobierno de Cantabria. Personal y SVO del matadero de Guarnizo. Cooperativa Agrocantabria. Programa DOC-INIA-CCAA 2008 (Emma Serrano).

\section{BIBLIOGRAFÍA}

\section{AOAC 1990. AOAC (Ed.) Official Methods of Analysis 15 th. AOAC.} Virginia.

Brandsstetter, A.M.; Picard, B. and Geay, Y. 1998. Muscle fibre characteristics in four muscles of growing male cattle. I. Effect of castration and feeding level. Livest Prod Sci, 53: 25-36.

Casasús, I.; Alberti, P.; Joy M.; Ripoll, G. y Blanco, M. 2011 . Influencia del nivel de suplementación sobre los rendimientos y características de la canal y la carne de terneros de raza Parda de Montaña cebados en pastoreo. Actas de las XIV Jornadas sobre Producción Animal, Zaragoza. pp. 61-63. http://www.aida-itea.org/aida-itea/files/jornadas/2011/comunicaciones/2011_SGEG_17.pdf. (19/2/2016).

Crouse, J.D.; Seideman, S.C. and Cross, H.R. 1983. The effects of carcass electrical stimulation and cooler temperature on the quality and palatability of bull and steer beef. J Anim Sci, 56: 81-90.
Humada, M.J.; Sañudo, C.; Cimadevilla, C. y Serrano, E. 2013. Efecto del sistema de producción y la edad de sacrificio sobre parámetros productivos, calidad de la canal y rendimiento económico de la producción de terneros y añojos de raza Tudanca. ITEA-Información Técnica Económica Agraria, 109: 183-200.

Humada, M.J.; Sañudo, C. and Serrano, E. 2014. Chemical composition, vitamin $\mathrm{E}$ content, lipid oxidation, color and cooking losses in meat from Tudanca bulls finished on semi-extensive or intensive systems and slaughtered at 12 or 14 months. Meat Sci, 96: 908-915.

INRA. 2007. Alimentation des bovins, ovins et caprins. Quae éditions. Versailles.

Knight, T.W.; Cosgrove, G.; Death, A. and Anderson, C. 1999. Effect of interval from castration of bulls to slaughter on carcass and meat quality. New Zeal J Agr Res, 42: 269-277.

Knight, T.W.; Cosgrove, G.; Death, A.; Anderson, C. and Fisher, A.D. 2000. Effect of method of castrating bulls on their growth rate and liveweight. New Zeal J Agr Res, 43: 187-192.

Mach, N.; Bach, A.; Realini, C.E.; Font i Furnols, M.; Velarde, A. and Devant, M. 2009. Burdizzo pre-pubertal castration effects on performance, behaviour, carcass characteristics and meat quality of Holstein bulls fed high-concentrate diets. Meat Sci, 8: 329-334.

Monteiro, A.C.G.; Navas, D.R. and Lemos, J.P.C. 2014. Effects of castration and time-on-feed on Mertolenga breed beef quality. Animal, 8: 675-682.

Ouali, A. 1992. Proteolytic and physicochemical mechanisms involved in meat texture development. Biochimie, 74: 251-265.

Sanz, A.; Ripoll, G.; Blasco, I.; Álvarez-Rodríguez, J. y Albertí, P. 2011. Potencial productivo de la raza Serrana de Teruel. Resultados preliminares. Arch Zootec, 60: 377-380.

SPSS 17.0. 2008. User's guide. SPSS Inc. Chicago.

Van Soest, P.J.; Robertson, J.B. and Lewis, B.A. 1991. Methods for dietary fiber, neutral detergent fiber and nonstarch polysaccharides in relation to animal nutrition. J Dairy Sci, 74: 3583-3597. 